\title{
Socio-Affective Computing
}

\author{
Volume 3
}

\section{Series Editor}

Amir Hussain, University of Stirling, Stirling, UK

\section{Co-Editor}

Erik Cambria, Nanyang Technological University, Singapore 
This exciting Book Series aims to publish state-of-the-art research on socially intelligent, affective and multimodal human-machine interaction and systems. It will emphasize the role of affect in social interactions and the humanistic side of affective computing by promoting publications at the cross-roads between engineering and human sciences (including biological, social and cultural aspects of human life). Three broad domains of social and affective computing will be covered by the book series: (1) social computing, (2) affective computing, and (3) interplay of the first two domains (for example, augmenting social interaction through affective computing). Examples of the first domain will include but not limited to: all types of social interactions that contribute to the meaning, interest and richness of our daily life, for example, information produced by a group of people used to provide or enhance the functioning of a system. Examples of the second domain will include, but not limited to: computational and psychological models of emotions, bodily manifestations of affect (facial expressions, posture, behavior, physiology), and affective interfaces and applications (dialogue systems, games, learning etc.). This series will publish works of the highest quality that advance the understanding and practical application of social and affective computing techniques. Research monographs, introductory and advanced level textbooks, volume editions and proceedings will be considered.

More information about this series at http://www.springer.com/series/13199 
Seng-Beng Ho

\section{Principles of Noology}

Toward a Theory and Science of Intelligence 


\author{
Seng-Beng Ho \\ Social and Cognitive Computing, \\ Institute of High Performance Computing \\ Agency for Science, Technology \\ and Research (A*STAR) \\ Singapore, Singapore
}

2009-2014

Temasek Laboratories

National University of Singapore

Singapore, Singapore

ISSN 2509-5706

Socio-Affective Computing

ISBN 978-3-319-32111-0

ISBN 978-3-319-32113-4 (eBook)

DOI 10.1007/978-3-319-32113-4

Library of Congress Control Number: 2016943131

(C) Springer International Publishing Switzerland 2016

This work is subject to copyright. All rights are reserved by the Publisher, whether the whole or part of the material is concerned, specifically the rights of translation, reprinting, reuse of illustrations, recitation, broadcasting, reproduction on microfilms or in any other physical way, and transmission or information storage and retrieval, electronic adaptation, computer software, or by similar or dissimilar methodology now known or hereafter developed.

The use of general descriptive names, registered names, trademarks, service marks, etc. in this publication does not imply, even in the absence of a specific statement, that such names are exempt from the relevant protective laws and regulations and therefore free for general use.

The publisher, the authors and the editors are safe to assume that the advice and information in this book are believed to be true and accurate at the date of publication. Neither the publisher nor the authors or the editors give a warranty, express or implied, with respect to the material contained herein or for any errors or omissions that may have been made.

Printed on acid-free paper

This Springer imprint is published by Springer Nature

The registered company is Springer International Publishing AG Switzerland 
To Leonard Uhr 



\section{Preface}

Despite the tremendous progress made in the past many years in cognitive science, which includes sub-disciplines such as neuroscience, psychology, artificial intelligence (AI), linguistics, and philosophy, there has not been an attempt to articulate a principled and fundamental theoretical framework for understanding and building intelligent systems. A comparison can be made with physics, which is a scientific discipline that led to the understanding of the physical universe and the construction of various human artifacts. The feats we have achieved through physics are indeed incredible: from mapping the cosmos to the end of space and time to the construction of towering skyscrapers and rockets that took human beings to the moon. Physics provides the principles and theoretical framework for these incredible feats to be possible. Is it possible to construct a similar framework for the field of cognitive science?

Whether intelligent systems are those that exist in nature, such as animals of all kinds, or the various kinds, of robots that human beings are trying to construct, they are all autonomous intelligent agents. Moreover, animals are adaptive autonomous intelligent agents (AAIAs), and the robots that human beings construct are also intended to be adaptive, though we have been falling short of the achievement of nature in this regard so far.

Interestingly, neuroscientists and psychologists do not seem to construe their respective disciplines as attempting to uncover the nature and principles of intelligence per se nor do they often characterize the systems they study as AAIAs. Neuroscientists are primarily concerned with uncovering the neural mechanisms in various human and animal brain subsystems such as the perceptual systems, affective systems, and motor systems, and how these various subsystems generate certain behaviors. Psychologists also attempt to understand human and animal behaviors through behavioral experiments and fMRI. But it is not just behavior per se but intelligent behavior that the various animals and humans exhibit that improve their chances of survival, allow them to satisfy certain internal needs, etc. They are also adaptive and autonomous intelligent agents. Hence, the numerous experimental works conducted in the fields of neuroscience and psychology so far 
have not benefitted from or been guided by a principled theoretical framework that characterizes adequately the systems that they are studying.

On the other hand, AI has been concerned with constructing AAIAs (also called "robots") right from the beginning. However, the shortcoming of AI at its current state of development is that the major "successes" are in creating specialized intelligent systems - systems such as the Deep Blue chess playing system that can beat human chess masters, the Watson questioning-answering system that can outperform human opponents, the upcoming autonomous vehicles (such as the Google self-driving car) that can drive safely on the roads, etc. But some researchers in the AI community do attempt to strive toward constructing general AAIAs in the long run. This is reflected in the emergence of a field called artificial general intelligence (AGI), though ironically, AI, in its very inception, was meant to be AGI to begin with.

An interesting question arises concerning human-constructed intelligent systems. Can a system that is not mobile in itself but that has remote sensors and actuators benefit from the principles guiding adaptive autonomous intelligent systems? The answer is yes, and we can think of the system as a kind of "static" robot. Because, with remote sensors and actuators, it can achieve the same effect as in the case of an AAIA as it learns about the environment through observation and interaction, and enriches its knowledge and changes its future behavior accordingly.

AGI can certainly learn from the rest of cognitive science. For example, in traditional AI research, the issues of motivation and emotion for an adaptive intelligent system, which provide the major driving force behind the system and are hence critical in its adaptive behavior, are never discussed (a scan of the major current textbooks in AI would reveal that these terms do not even exist in the index), while these are often studied extensively in neuroscience and psychology. The issues of affective processes, however, are gaining some attention recently in the field of AGI/AI.

In this book we therefore set out toward a more systematic and comprehensive understanding of the phenomenon of intelligence, thus providing a principled theoretical framework for AAIAs. The methodology is similar to that of AGI/AI, in which representational mechanisms and computational processes are laid out clearly to elucidate the concepts and principles involved. This is akin to the quantitative and mathematical formulation of the basic principles of physics that embodies rigorous understanding and characterization of the phenomena involved. There are two advantageous to this approach: on the one hand, these mechanisms translate to directly implementable programs to construct artificial systems; on the other hand, these mechanisms would direct neuroscientists and psychologists to look for corresponding mechanisms in natural systems. Because of the relatively detailed specifications of the representational mechanisms and computational processes involved, they may guide neuroscientists and psychologists to understand brain and mental processes at a much higher resolution, and also understand them in the context of AAIAs, which is a paradigm that is currently lacking in these fields.

The representational mechanisms and computational processes employed in this book are not strange to people in the field of AI: predicate logic representations, 
search mechanisms, heuristics, learning mechanisms, etc. are used. However, they are put together in a new framework that addresses the issues of general intelligent systems. Some novel computational devices are introduced, notably the idea of rapid effective causal learning (which provides a rapid kind of learning subserving critical intelligent processes), learning of scripts (which provides a foundation for knowledge chunking and rapid problem solving), learning of heuristics (which enhances traditional AI's methodology in this regard which often employs heuristics that are built-in and not learned in a typical problem solving situation), semantic grounding (which lies at the heart of providing the mechanisms for a machine to "really understand" the meaning of the concepts that it employs in various thinking and reasoning tasks), and last but not least, the computational characterizations of motivational and affective processes that provide purposes and drives for an AAIA and that are critical components in its adaptive behavior.

From the point of view of identifying fundamental entities for the phenomenon of intelligence (much in the same spirit in physics of identifying fundamental particles and interactions from which all other phenomena emerge), two ideas stand out. One is atomic spatiotemporal conceptual representations and their associated processes, which provide the ultimate semantic grounding mechanisms for meaning, and the other is the script, which encodes goal, start state, and solution steps in one fundamental unit for learning and rapid problem solving operations.

We think it necessary to introduce the term "noology" (pronounced \nō-'ä-lə-jē in the same vein as "zoology") to designate the principled theoretical framework we are attempting to construct. Noology is derived from the Greek word "nous" which means "intelligence." The Merriam-Webster dictionary defines noology as "the study of mind: the science of phenomena regarded as purely mental in origin." The function of noology - a theoretical framework for and the science of intelligence is like the function of physics. It provides the principled theoretical framework for, on the one hand, the understanding of natural phenomena (namely all the adaptive autonomous intelligent systems (AAISs) that cognitive scientists are studying), and on the other, the construction of artificial systems (i.e., robots, all kinds of autonomous agents, "intelligent systems," etc., that are the concerns of AGI/AI).

In cognitive science, it has been quite a tradition to use "cognitive systems" to refer to the "brain" systems that underpin the intelligent behavior of various kinds of animals. However, as has been emphasized in a number of works by prominent neuroscientists such as Antonio Damasio and Edmund Roll, cognition and emotion are inseparable and together they drive intelligent behaviors as exhibited by AAISs. Therefore, "noological systems" would be a more appropriate characterization of systems such as these.

We do not pretend to have all the answers to noology. Therefore, the subtitle of this book is "toward a theory and science of intelligence." But we believe it sets a new stage for this new, and at the same time old, and exciting endeavor.

For readers familiar with the computational paradigm (e.g., AI researchers), it is recommended that they jump ahead to take a look at Chaps. 6 and 7 and perhaps also 8 and 9, where our paradigm is applied to solve some problems that would typically be encountered by AI systems, before returning to start from the beginning 
of the book. Our method derives "noologically realistic" solutions that use very different processes than that of traditional AI methods. After having a rough idea of what we are trying to achieve, the purpose of the relatively extensive groundwork covered in the first few chapters would then be clearer.

Singapore, Singapore

Seng-Beng Ho 


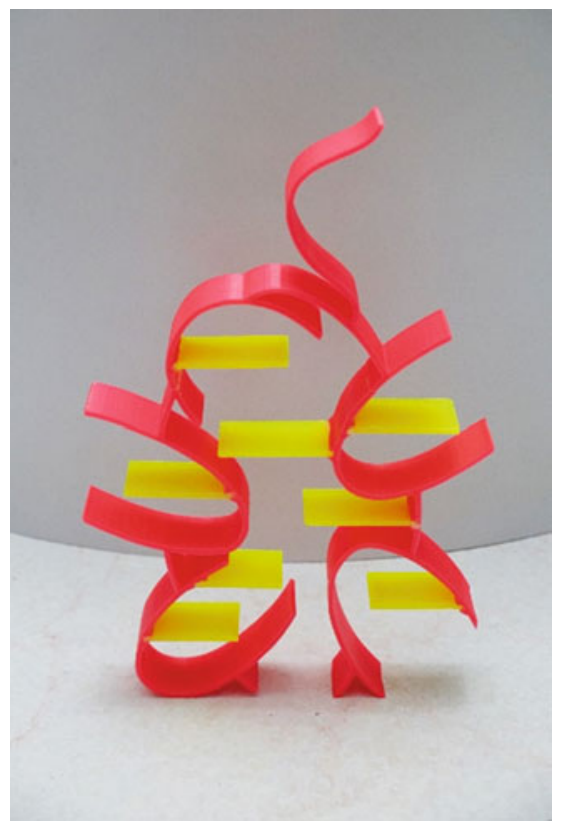

A Celebration of Concavity

Shelf: Designed by Seng-Beng Ho Design Registration No. D2014/888/A

"Science and art in the pursuit of beauty, and hence, truth" 



\section{Acknowledgements}

In a sense the beginning of this book is lost in the author's personal mist of time. My formal journey on the path to understand intelligence began in the Fall of 1981 when I started my graduate school program at the University of WisconsinMadison. Because the effort culminating in this book was an incessant effort from more than 30 years ago, some acknowledgements are due to some of the people from that time.

My Ph.D. supervisor was the late Professor Leonard Uhr who was one of the pioneers in artificial intelligence, as reflected by the fact that one of his important works is collected in the book, Computers and Thought, published in 1963, 8 years after John McCarthy coined the term "artificial intelligence (AI)." One of the editors of the book was Edward Feigenbaum of Stanford University, a wellknown researcher in AI. Leonard Uhr's research was a little off "mainstream AI" and hence he was not as well-known as, say, Edward Feigenbaum or Marvin Minsky of MIT in the field of AI.

However, my thanks are due to Leonard Uhr for allowing me the freedom to think outside the mainstream research trend in AI of that time, which allowed me to address critical issues ignored by others. This constituted my Ph.D. thesis work of that time and it set the stage for my subsequent pursuit in understanding intelligence in the same spirit, and which then led to this book of today. Thanks are also due to Chuck Dyer, Josh Chover, Gregg Oden, Lola Lopes, Robin Cooper, and Berent Enc, who were professors of that time who interacted with me in one way or another and who were equally encouraging and supportive of my unique focus on issues related to intelligent systems.

The intellectual origin of this book can be traced back to more than 30 years ago but the bulk of the work contained herein was carried out in the Temasek Laboratories of the National University of Singapore in the period 2011-2014. I wish to thank the Head of the Cognitive Science Programme at Temasek Laboratories, Dr. Kenneth Kwok, who, like the professors before him, fully supported my research that addressed critical and important issues not attended to by other AI researchers. 
All the computer simulations in the book and the associated graphical renderings were done by Fiona Liausvia. My sincerest thanks to her for a fantastic job done.

Erik Cambria's work on sentic computing was an inspiration. My own approach to computational affective processes started on a different track, but knowing about Erik's work and exchanging ideas with him provided me with the confidence that we are moving in the right direction with regards to a more complete computational characterization of intelligent systems.

My immediate family has always provided great intellectual companionship. Thanks are absolutely due to them for keeping me intellectually alive all through the years.

Singapore, Singapore

Seng-Beng Ho

January 1, 2016 


\section{Contents}

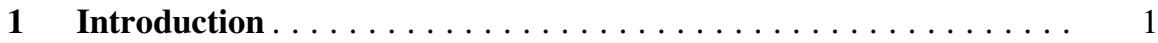

1.1 The Core Functional Blocks of a Noological System . . . . . . . 6

1.2 Basic Considerations Motivating a Noological Processing Architecture . . . . . . . . . . . . . . . . . 8

$1.3 \quad$ A Basic Noological Processing Architecture . . . . . . . . . . 15

1.4 The Bio-noo Boundary and Internal Grounding . . . . . . . 20

1.5 Motivation/Goal Competition and Adaptation, and Affective Learning . . . . . . . . . . . . . . . . . . 25

1.6 External and Internal Grounding: Breaking the Symbolic

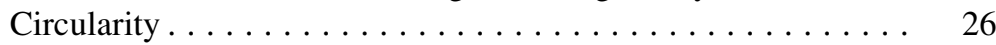

1.7 Perception, Conception, and Problem Solving . . . . . . . . . . 29

1.8 Addressing a Thin and Deep Micro-environment

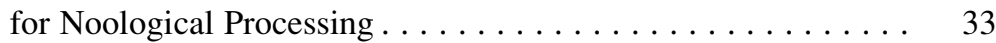

1.9 Summary of the Basic Noological Principles . . . . . . . . . . 36

Problem.................................. 37

References............................... 37

2 Rapid Unsupervised Effective Causal Learning . . . . . . . . . . 41

$2.1 \quad$ Bayesian Causal Inference and Rapid Causal Learning . . . . . . . 43

2.2 On Effective Causality..................... 44

2.3 Opportunistic Situation and Rapid Cause Recovery . . . . . . . . 48

2.4 Diachronic and Synchronic Causal Factors in Rapid Causal Learning. . . . . . . . . . . . . . . . . . . . . . 51

2.5 Desperation, Generalization, and Rule Application........ . 59

2.6 Application: Causal Learning Augmented Problem Solving Search Process with Learning of Heuristics . . . . . . . . . . 61

2.6.1 The Spatial-Movement-to-Goal (SMG) Problem . . . . . 61

2.6.2 Encoding Problem Solution in a Script: Chunking . . . . 67

2.6.3 Elemental Actions and Consequences: Counterfactual Script ....................... 75 
2.6.4 Maximum Distance Heuristic for Avoidance of Anti-goal . . . . . . . . . . . . . . . . 79

2.6.5 Issues Related to the SMG Problem and the Causal Learning Augmented Search Paradigm . . . . . . . . 80

2.7 Toward a General Noological Forward Search

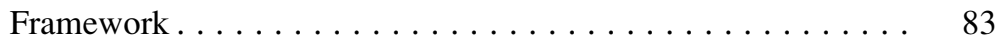

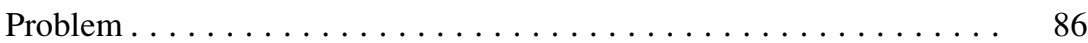

References............................ 87

3 A General Noological Framework . . . . . . . . . . . . . . . . . . 89

3.1 Spatial Movement, Effort Optimization,

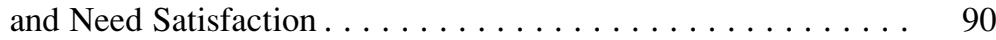

3.1.1 MOVEMENT-SCRIPT with Counterfactual

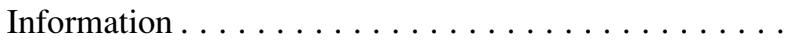

3.2 Causal Connection Between Movement

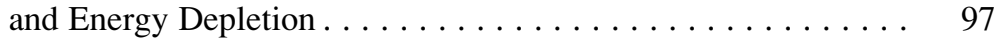

3.3 Need and Anxiousness Competition . . . . . . . . . . . . . . . . . . . . . 99

3.3.1 Affective Learning . . . . . . . . . . . . . . . . . 103

3.4 Issues of Changing Goal and Anti-goal . . . . . . . . . . . . . . . . . . . . . . . . . 104

3.5 Basic Idea of Incremental Knowledge Chunking . . . . . . . . . . 107

3.5.1 Computer Simulations of Incremental Chunking . . . . . 111

3.6 Motivational Learning and Problem Solving . . . . . . . . . . . . 119

3.7 A General Noological Processing Framework . . . . . . . . . . . . 122

3.8 Neuroscience Review . . . . . . . . . . . . . . . . . . . . . . . . . . . 124

3.8.1 The Basal-Ganglionic-Cortical Loops . . . . . . . . . . 125

3.8.2 The Cerebellar-Cortical Loops . . . . . . . . . . . . . . . . 129

3.8.3 The Houk Distributed Processing Modules

(DPMs) . . . . . . . . . . . . . . . . . . . . . . 129

3.8.4 The Computational Power of Recurrent

3.8.5 Overall General Brain Architecture . . . . . . . . . . . . . 134

3.9 Mapping Computational Model to Neuroscience Model . . . . . . 136

3.10 Further Notes on Neuroscience . . . . . . . . . . . . . . . . . 138

3.10.1 The Locus of Memory Is Not the Synapse . . . . . . . . 139

3.10.2 Function of the Prefrontal Cortex

and the Entire Brain . . . . . . . . . . . . . . . . 140

Problems . . . . . . . . . . . . . . . . . . . . . . . . . . 141

References............................ 141

4 Conceptual Grounding and Operational Representation . . . . . . . . 145

4.1 Ground Level Knowledge Representation and Meaning . . . . . . 146

4.2 An Operational Representational Scheme for Noological

Systems . . . . . . . . . . . . . . . . . . . . 152

4.3 Operational Representation . . . . . . . . . . . . . . . . . 153

4.3.1 Basic Considerations . . . . . . . . . . . . . 153

4.3.2 Existential Atomic Operators . . . . . . . . . . 158 
4.3.3 Movement-Related Atomic Operators . . . . . . . . . . 160

4.3.4 Generation of Novel Instances of Concepts . . . . . . . . 162

4.3.5 Example of 2D Movement Operations . . . . . . . . . . 162

4.3.6 Characteristics of Operational Representation . . . . . . 163

4.3.7 Further Issues on Movement Operators . . . . . . . . . . 164

4.3.8 Atomic Operational Representations of Scalar and General Parameters . . . . . . . . . . . . . . . 171

4.3.9 Representing and Reasoning About Time . . . . . . . . . 173

4.3.10 Representation of Interactions . . . . . . . . . . . . . . . . 174

4.4 Issues on Learning . . . . . . . . . . . . . . . . . . . . . . . . . . . . 183

4.5 Convergence with Cognitive Linguistics . . . . . . . . . . . . . 185

4.6 Discussion . . . . . . . . . . . . . . . . . . . . . . . . . . 187

Problems . . . . . . . . . . . . . . . . . . . . . . . . . . 188

References.......................... 188

5 Causal Rules, Problem Solving, and Operational

Representation . . . . . . . . . . . . . . . . . . . . . . 191

$5.1 \quad$ Representation of Causal Rules . . . . . . . . . . . . . . . 191

5.1.1 Materialization, Force, and Movement . . . . . . . . . 192

5.1.2 Reflection on Immobile Object, Obstruction, and Penetration . . . . . . . . . . . . . . . . . 196

5.1.3 Attach, Detach, Push, and Pull . . . . . . . . . . . . 198

5.2 Reasoning and Problem Solving . . . . . . . . . . . . . . . . 198

5.2.1 Examples of Problem Solving Processes . . . . . . . . . 201

5.2 .2 Incremental Chunking . . . . . . . . . . . . . . . 205

5.2.3 A More Complex Problem . . . . . . . . . . . . . . . 208

5.3 Elemental Objects in 2D: Representation and Problem

Solving . . . . . . . . . . . . . . . . . . 210

5.4 Natural Language, Semantic Grounding, and Learning to Solve Problem Through Language . . . . . . . . . . . . . . 213

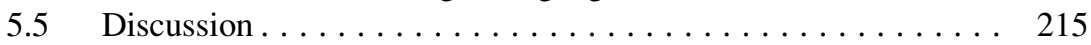

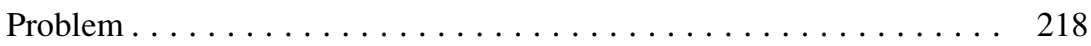

References......................... 218

6 The Causal Role of Sensory Information . . . . . . . . . . . . . . . 221

6.1 Information on Material Points in an Environment . . . . . . . . 222

6.2 Visual Information Provides Preconditions for Causal Rules . . . . . . . . . . . . . . . . . . . . 226

6.3 Inductive Competition for Rule Generalization . . . . . . . . . . 230

6.4 Meta-level Inductive Heuristic Generalization . . . . . . . . . . . . 234

6.5 Application to the Spatial Movement to Goal with Obstacle (SMGO) Problem . . . . . . . . . . . . . . . . . . . . 238

6.5.1 Script and Thwarting of Script . . . . . . . . . . 241

6.5.2 Recovery from Script Thwarting Through Causal Reasoning. . . . . . . . . . . . . . . . . . . . . . 244 
6.6 A Deep Thinking and Quick Learning Paradigm . . . . . . . . . 280

Problem . . . . . . . . . . . . . . . . . . . . . . . . . . . . . 282

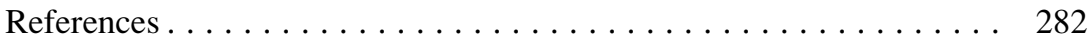

7 Application to the StarCraft Game Environment . . . . . . . . . . . 283

7.1 The StarCraft Game Environment . . . . . . . . . . . . . . . . . . . . 284

7.1.1 The Basic Scripts of the StarCraft Environment . . . . . 286

7.2 Counterfactual Scripts and Correlation Graphs of Parameters . . . . . . . . . . . . . . . . . . . . . . . . . . . . 291

7.3 Desperation and the Exhaustiveness of Observations and Experiments . . . . . . . . . . . . . . . . . . . . . 299

7.4 Rapid Learning and Problem Solving in StarCraft . . . . . . . . 300

7.4.1 Causal Learning to Engage/Attack Individual Enemy Agents . . . . . . . . . . . . . . . . . 301

7.4.2 Affective Competition and Control: Anxiousness Driven Processes . . . . . . . . . . . . . . . . . . . . 313

7.4.3 Causal Learning of Battle Strategies . . . . . . . . . . . 317

7.5 Learning to Solve Problem Through Language . . . . . . . . . . . 340

7.6 Summary ......................... 341

References... . . . . . . . . . . . . . . . . . . . . . . . . . 342

8 A Grand Challenge for Noology and Computational Intelligence . . 343

8.1 The Shield-and-Shelter (SAS) Micro-environment . . . . . . . . . . 344

8.1.1 Basic Considerations . . . . . . . . . . . . . . . 344

8.1.2 Activities in the Micro-environment . . . . . . . . . 345

8.1.3 Further Activities and Concepts . . . . . . . . . . . 350

8.2 The Generality of the SAS Micro-environment . . . . . . . . . . 351

8.3 The Specifications of the SAS Micro-environment

Benchmark . . . . . . . . . . . . . . . . . . . 354

$8.4 \quad$ Conclusion . . . . . . . . . . . . . . . . . . . . . . . . . . 356

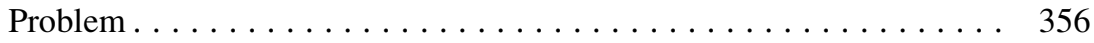

References.......................... 356

$9 \quad$ Affect Driven Noological Processes . . . . . . . . . . . . . . . . . . . . . 359

9.1 Learning and Encoding Knowledge on Pain-Causing

Activities . . . . . . . . . . . . . . . . . . . . . 360

9.2 Anxiousness Driven Noological Processes . . . . . . . . . . . . . . 364

9.3 Solutions to Avoid Future Negative Outcome . . . . . . . . . . . 367

9.3.1 Causal Reasoning to Identify the Cause of Negative Outcome . . . . . . . . . . . . . . 368

9.3.2 Identifying a Method to Remove the Cause of Negative Outcome . . . . . . . . . . . . . 370

9.3.3 A Second Method to Remove the Cause of Negative Outcome . . . . . . . . . . . . . . . 377

9.4 Further Projectile Avoidance Situations and Methods . . . . . . . 383

9.4.1 Structure Construction and Neuroticism Driven Processes.................... 386 
9.5 Summary and Future Investigations . . . . . . . . . . . . . 387

Problems . . . . . . . . . . . . . . . . . . . . . . . . . . . . . . 389

References.......................... 389

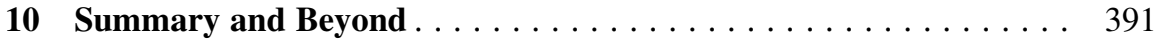

10.1 Scaling Up to the Complex External Environment . . . . . . . . . 394

10.2 Scaling Up to the Complex Internal Environment . . . . . . . . . 399

10.3 Explicit Representations of Learnable Mental Processes . . . . . . 402

10.4 Perception, Semantic Networks, and Symbolic Inference . . . . . 406

10.5 Personality, Culture, and Social Situations . . . . . . . . . . . . 408

10.6 Comparisons with Methods in AI . . . . . . . . . . . . . . 408

10.6.1 Connectionism vs Symbolic Processing . . . . . . . . . . . 409

10.6.2 Effective Causal Learning vs Reinforcement and Other Learning Mechanisms . . . . . . . . . . . . 411

10.6.3 Deep Learning vs Deep Thinking and Quick Learning . . . . . . . . . . . . . . . . . . . . 413

10.7 A Note on Biology . . . . . . . . . . . . . . . . . . . . . 413

References......................... 415

Appendices . . . . . . . . . . . . . . . . . . . . . . . . . . . . 419

Appendix A: Causal vs Reinforcement Learning . . . . . . . . . . . . . . 419

Appendix B: Rapid Effective Causal Learning Algorithm . . . . . . . . 421

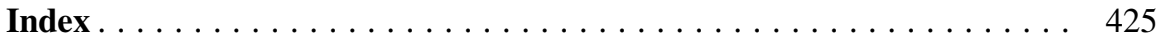

